\title{
A Patient with Severe Cervicofacial Subcutaneous Emphysema Associated with Munchausen's Syndrome: A Case Report
}

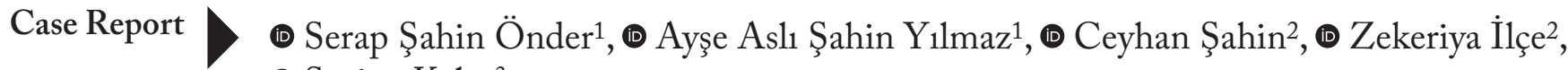 \\ (1) Sevinç Kalın ${ }^{3}$ \\ ${ }^{1}$ Department of Otolaryngology, University of Health Sciences Turkey, Ümraniye Training and Research Hospital, \\ İstanbul, Turkey \\ ${ }^{2}$ Department of Pediatric Surgery, University of Health Sciences Turkey, Ümraniye Training and Research Hospital, \\ İstanbul, Turkey \\ ${ }^{3}$ Department of Radiology and Imaging, University of Health Sciences Turkey, Ümraniye Training and Research \\ Hospital, İstanbul, Turkey
}

\section{Abstract \\ ORCID ID of the authors: \\ S.Ş.O. 0000-0002-3576-0953; \\ A.A.S.Y. Y. 0000-0002-7846-9453; \\ C.S. $0000-0003-3101-3915$ \\ Z.i. 0000-0002-3473-5051; \\ S.K. 0000-0001-9417-2847. \\ Cite this article as: Sahin Önder S, Sahin YIIImaz AA, Sahin C, Ilkse Z, Kalın S. A Patient with Severe Cervicofacial Subcutaneous Emphysema Associated with Munchausen's Syndrome: A Case Report. Turk Arch Otorhinolaryngol 2021; 59(3):230-3. \\ Corresponding Author: \\ Serap şahin Önder; serapsahinonder@gmail.com \\ Received Date: 29.03.2021 Accepted Date: 08.06 .2021 \\ Content of this journal is licensed under a Creative Commons Attribution 4.0 International License. Available online at www.turkarchotolaryngol.net (c) (i) (8)}

Subcutaneous cervicofacial emphysema is a rare and life-threatening condition that results from various causes. In this report, we documented a case of a patient with severe subcutaneous cervicofacial emphysema a condition that falls under the umbrella of Munchausen's syndrome and discussed the workup of this patient. Thorough diagnostic investigations seeking the etiology of the condition proved unsuccessful. When faced with cases of recurring subcutaneous cervicofacial emphysema, where the root cause remains ambiguous a diagnosis of Munchausen's Syndrome should be considered.

Keywords: Munchausen syndrome, subcutaneous emphysema, neck, pediatric otorhinolaryngology, case report

\section{Introduction}

Munchausen's Syndrome (MS) was initially documented by Asher in 1951 (1). Sufferers of the syndrome are characterized as being wholly conscious persons who falsify physical or psychological signs in order to appear sick. Self-induced subcutaneous emphysema, a rare form of MS was first observed by Gershwin in 1971 (2). The following report outlines a case of an adolescent with MS who required aggressive management due to multiple subcutaneous cervicofacial emphysema.

\section{Case presentation}

The patient, a 16-year-old female complained of recurrent instances of swelling on her neck, chest wall, and left arm. Her medical history included two surgeries under general anesthesia with orotracheal intubation; septoplasty (one 
year prior) and nasal septal perforation repair (two months prior). After septoplasty, she had recurrent epistaxis episodes for six months which were treated with nasal packings when needed. She eventually had a nasal septal perforation and was referred to another tertiary care hospital by her primary surgeon. There she underwent her second surgery under general anesthesia which involved septal perforation repairment. After this surgery she started having epistaxis episodes which were treated with cauterization and nasal packing.

One month following the septal perforation repairment surgery, the patient suffered her first episode of subcutaneous emphysema on her left arm, chest, and neck. The chest computed tomography (CT) revealed pneumothorax and subcutaneous emphysema. The pneumothorax was treated with chest tube insertion. The patient underwent an endoscopic examination of head and neck, as well as bronchoscopy to discover the etiology of pneumothorax and subcutaneous emphysema, which revealed normal findings. The subcutaneous emphysema regressed slowly and disappeared.Two months after the firstepisode of emphysema, she was referred to the pediatric surgery department at our hospital with swelling on her neck and on her upper part of the chest. She underwent a detailed investigation, involving several consultations with otolaryngology, dentistry, pediatric gastroenterology, infectious disease, allergy and dermatology departments, however, these provided little clarity into the patient's condition. All initial laboratory tests (erythrocyte sedimentation rate, blood count, urine tests, liver and kidney function tests) and cultures (blood, nasal cavities, nasopharynx,

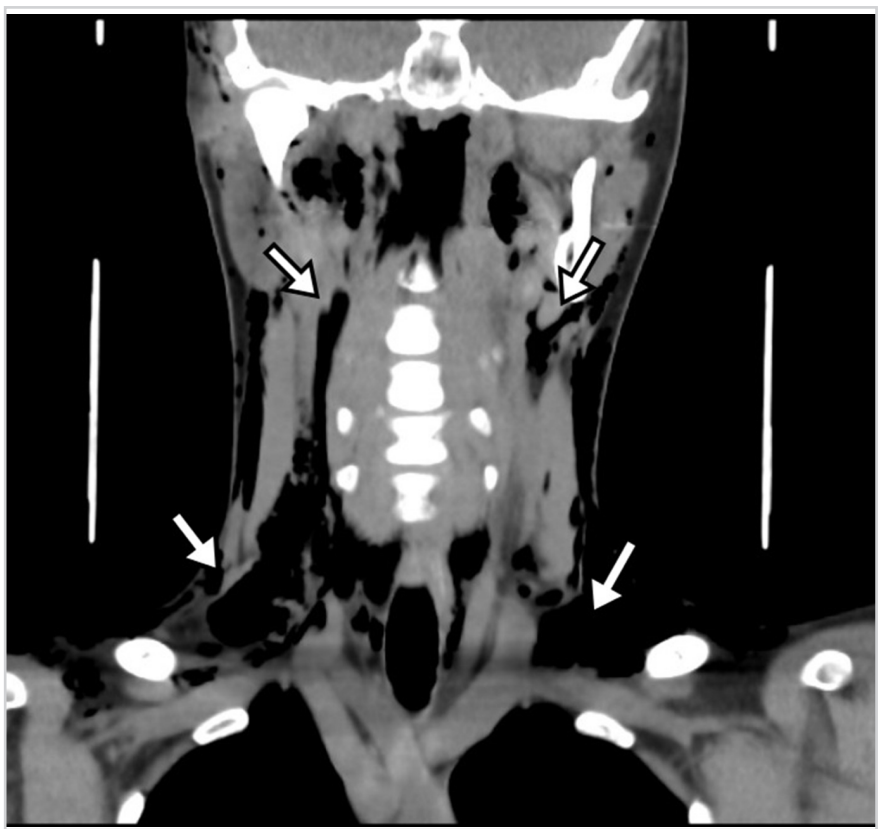

Figure 1. Computed tomography scan of the patient reveals the presence of extensive air (arrows) within the fascial planes of the head and neck lung aspiration and throat for bacteria) were essentially normal. Oral examination was negative for perforated ulcers or dental abscess, and ear-throat-nose examination did not unearth any irregularities. CT scan of the chest excluded pneumomediastinum and other pulmonary pathologies that could result in subcutaneous emphysema. A subsequent endoscopy did not reveal any irregularities. The patient had spontaneous resolution of symptoms and was discharged from the hospital. Two months following her discharge the patient presented with bilateral massive periorbital edema and subcutaneous emphysema on her neck. She was hospitalized and transferred to our department. Panendoscopy of head and neck and bronchoscopy were performed for a second time and the examinations were inconclusive. CT of the chest and neck revealed subcutaneous emphysema on her neck and face (Figure 1). The patient was subjected to a second course of laboratory tests and with cultures being taken again and similarly to the first round the results did not unearth pathological signs.

After a detailed inquiry into her previous surgeries, the parents consented our team to contact the surgeon who had previously operated her for nasal septal perforation. The previous surgeon mentioned that he was suspicious that the recurrent epistaxis episodes and the resulting nasal septal perforation, as well as the recurrent epistaxis episodes after the perforation repair, could be due to self-induced digital trauma since no underlying hematologic condition was detected.

With the etiology proving elusive, self-injected air was deliberated as a potential diagnosis and the psychiatry department was consulted. Mounting suspicion that the illness may be self-inflicted meant that a stricter watch of the patient was required. The risk posed by misdiagnosis and to prevent the administering of unnecessary surgical procedures hospital staff carried out a search of the patient's belongings. The search uncovered four syringes as well as blood-stained needles. The patient was referred to the department of psychiatry. Based on her recurrent negative clinical and laboratory evaluations and evidence found in the search, a diagnosis of MS was suspected which would indicate her symptoms resulted from self-abusive behavior and would explain her sustained exaggeration nature of said symptoms. When the patient was confronted with the evidence outlining the justification for her diagnosis, she became agitated and uncooperative, finally discharging herself from the hospital against her caregivers' wishes. The patient was discharged leaving behind a high medical bill consisting of 40 days of hospitalization, two surgeries under general anesthesia and eight CT scans of head, neck and chest region. Monthly follow-up appointments with psychiatry outpatient continued for a duration of six months and these confirmed the diagnosis of MS. 


\section{Discussion}

There are few reports in literature that discuss subcutaneous emphysema arising from MS. Koufagued et al. (3) reported a case who presented with subcutaneous emphysema of the shoulder girdle and the right arm. The treatment included a wide surgical debridement, hyperbaric oxygen therapy, and parenteral antibiotic therapy. Tosun et al. (4) recorded an episode of subcutaneous cervicofacial emphysema that stemmed from MS. The patient underwent a tracheotomy to secure the airway. Yucel et al. (5) forwarded a case of an adolescent with orbital emphysema. The authors performed a tracheostomy, anteroposterior nasal packing. In total 41 consultants were involved in administering treatment to the patient across the eight months the patient was hospitalized. A case of a woman aged 36 who presented with swelling of left orbital, pain, vision loss, diplopia, fever, and prostration associated with MS was reported by Greene et al. (6). The common denominator in all these cases is recurrent emphysema, the cause of which could not be found, and the patients were diagnosed with MS. In the present case, the subcutaneous emphysema recurred three times, moreover, no etiological factors were found.

Studies into factitious disorder demonstrate the impact unnecessary investigations, treatments, and hospital admission have on the health care system as evidenced by our case (7).

The patient underwent several surgeries, consultations and radiological assessments that resulted in unnecessary exposure to general anesthesia and radiation, as well as a high medical bill.

The literature detailed cases involving children where the deception was discovered either because they were so obvious even after a brief consultation or because health care workers at the hospital carried out a search of the patient's belongings after considering all other diagnoses (8). In the present case, hospital staff carried out a search of the patient's belongings. The search uncovered four syringes as well as blood-stained needles giving rise to suspicions of MS.

A review on pediatric factitious illnesses confirmed the mean age of the children to be 13.9 years, with the range spanning years 8 to 18 . The authors noted that the average length of deception was shorter amongst the younger cohort of children that being (12.8 months) compared with the older group (18.9 months). Furthermore, older children, as well as adolescences' medical deceptions, included ingesting steroids leading to an onset of factitious Cushing's Syndrome or by injecting air and thereby causing subcutaneous facial emphysema. Evidence also points to the introduction of eggs and other foreign materials into their bladders to induce proteinuria as well as used tourniquets and feculent urine to cause swelling and wrist pain. In addition, at least $45 \%$ of older children stubbornly maintained their denial even when confronted with concrete evidence (8). In the present case, the adolescent did not own up even when presented with the evidence that was full proof.

\section{Conclusion}

MS offers a diagnostic predicament that requires increased medical and social attention. Furthermore, it is a condition that demands sustained research, and more effort should be made to spread awareness not only amongst the public at large but also to health care providers. Moreover, early recognition of MS is essential in order to restrict wastage of healthcare resources and reduce patient suffering. When a thorough diagnostic investigation fails to ascertain the cause for a patient's recurring symptoms, a diagnosis of MS should be considered.

Informed Consent: Informed consent form was obtained from the patient's parents.

Peer-review: Externally peer-reviewed.

Conflict of Interest: No conflict of interest was declared by the authors.

Financial Disclosure: The authors declared that this study received no financial support.

\section{Authorship Contributions}

Surgical and Medical Practices: S.Ş.Ö., A.A.Ş.Y., C.Ş., Z.İ., S.K., Concept: S.Ş.Ö., A.A.Ş.Y., C.Ş., Design: S.Ş.Ö., A.A.Ş.Y., Data Collection and/or Processing: S.Ş.Ö., A.A.Ş.Y., C.Ş., Z.İ., S.K., Analysis and/or Interpretation: S.Ş.Ö., A.A.Ş.Y., Literature Search: S.Ş.Ö., A.A.Ş.Y., C.Ş., Z.İ., S.K., Writing: S.Ş.Ö., A.A.Ş.Y.

\section{Main Points}

- If detailed diagnostic investigations fail to reveal the cause of subcutaneous emphysema, a diagnosis of MS should be considered.

- The correlation between anamnestic data and clinical and paraclinical examinations are essential for the diagnosis of MS.

- Early recognition of MS is paramount to spare the patient from needless and potentially harmful diagnostic tests as well as saving hospitals from unnecessary financial outlays.

\section{References}

1. Asher R. Munchausen's syndrome. Lancet 1951; 1: 339-41. [Crossref]

2. Gershwin ME, Gude JK, Petralli J. Factitious subcutaneous emphysema. Ann Intern Med 1971; 75: 585-7. [Crossref] 
3. Koufagued K, Chafry B, Benyass Y, Abissegue Y, Benchebba D, Bouabid S, et al. Munchausen syndrome revealed by subcutaneous limb emphysema: a case report. J Med Case Rep 2015; 18: 9: 172. [Crossref]

4. Tosun F, Ozer C, Akcam T, Gerek M, Yetiser S. A patient of severe cervicofacial subcutaneous emphysema associated with Munchausen's syndrome. J Craniofac Surg 2005; 16: 661-4. [Crossref]

5. Yucel EA, Keles N, Arami K, Orhan S, Deger K. Munchausen's syndrome: a case report with multiple symptoms. Otolaryngol Head Neck Surg 2001; 124: 595-7. [Crossref]
6. Greene D, Murr AH. Factitious orbital emphysema: an unusual presentation of Munchausen's syndrome. Otolaryngol Head Neck Surg 1998; 119: 512-4. [Crossref]

7. Caselli I, Poloni N, Ielmini M, Diurni M, Callegari C. Epidemiology and evolution of the diagnostic classification of factitious disorders in DSM-5. Psychol Res Behav Manag 2017; 10: 387-94. [Crossref]

8. Libow JA. Child and adolescent illness falsification. Pediatrics 2000; 105: 336-42. [Crossref] 